\title{
The Study of Pedestrian Flow Based on a Macroscopic Model
}

\author{
Yanqun Jiang* \\ Department of Mathematics \\ Southwest University of Science and Technology \\ Mianyang, China \\ jyq2005@mail.ustc.edu.cn
}

\author{
Shuguang Zhou \\ China Aerodynamics Research and Development Center \\ Mianyang, China
}

\begin{abstract}
In this paper, a macroscopic crowd model is developed to simulate pedestrian flow characteristics and walking behaviors in a facility scattered with an obstacle. Pedestrians are assumed to walk towards a desired destination deviating from the path of shortest travel time according to the environmental factors. Some preliminary numerical results demonstrate reasonable path choice strategy for pedestrians can reduce the chance of traffic congestion occurring.
\end{abstract}

Index Terms-Pedestrian flow; macroscopic model; path choice strategy; obstacle.

\section{INTRODUCTION}

Crowd dynamics is investigated generally by microscopic and macroscopic modeling methods. The microscopic modeling approach focuses on details of features of crowd movement and the behavior characteristics of each individual in a crowd. Models of this category, such as social force models [1,2] and cellular automaton models [3,4], increase computational complexity with the increase of the scale of individuals. However, they can reproduce individual behaviour observed in real emergency evacuation situation [1] The macroscopic modeling approach emphasizes the global crowd movement pattern and reduces complexity to fundamental principles of pedestrian flow. Models of this category, such as hydrodynamic models [5-10] described as a set of differential equations, are very useful in the simulation of physical movement of large scale crowds.

In this paper, a macroscopic crowd model is developed to simulate pedestrian flow walking in a facility scattered with an obstacle. Pedestrian walking behaviors and flow characteristics are formulated by the fundamental aspects as follows. First of all, pedestrians have a strong desire to continue their intended path of travel, e.g., the path of least time based on experience, which determines the main direction of pedestrian motion; secondly, they have an incentive to avoid environmental borders (e.g., walls and obstacles) in the moving process; thirdly, they tend to avoid the risk of collision, which introduces interactions among individuals. Therefore, pedestrians move towards the desired destination deviating from the path of least time according to the environmental factors.
The framework of the modeling algorithm is based on a cell-centered high-resolution finite volume discretization on an orthogonal grid and a total variation diminishing RungeKutta method for the time integration of semi-discrete equations [8]. Numerical simulation is carried out to investigate pedestrian flow characteristics and walking behaviors at the macroscopic level.

\section{PEDESTRIAN DYNAMIC MODEL}

We consider a crowd of pedestrians moving in a walking facility, $\Omega \in R^{2}$ (in $\mathrm{m}^{2}$ ). The sections of solid wall, entrance, exit are denoted by $\Gamma_{w}, \Gamma_{i}$, and $\Gamma_{o}$, respectively. Based on continuum dynamics, the conservation law for pedestrian flow is defined as follows.

$$
\left\{\begin{array}{c}
\frac{\partial \rho(\mathbf{x}, t)}{\partial t}+\nabla \square \rho(\mathbf{x}, t) \mathbf{v}(\mathbf{x}, t)=0 \\
\rho(\mathbf{x}, 0)=\rho_{0}(\mathbf{x})
\end{array}, \mathbf{x} \in \Omega, t \geq 0,\right.
$$

where $\rho(\mathbf{x}, t)$ (in $\mathrm{ped} / \mathrm{m}^{2}$ ) and $\mathbf{v}(\mathbf{x}, t)=U_{e} \mathbf{v}$ (in $\mathrm{m} / \mathrm{s}$ ) are the density and velocity of pedestrian flow, respectively. Here, $\boldsymbol{v}=\left(v_{1}(\mathbf{x}, t), v_{2}(\mathbf{x}, t)\right)$ is a unit vector representing the actual walking direction of pedestrians, and $U_{e}=U_{e}(\rho)$ is the magnitude of velocity or the speed-density relationship.

The description of direction of pedestrian motion, $\mathbf{v}$, is based on the following assumptions.

1. Pedestrians always try to reach the desired goal along the path of shortest travel time, which determines the main direction of pedestrian motion, in a familiar walking environment. Let $\Phi(\mathbf{x})$ be the minimum travel cost from $\mathbf{x} \in \Omega$ to $\mathbf{x}_{0} \in \Gamma_{o}$ (the exit) and the main direction of pedestrian motion, $\boldsymbol{v}^{1}(\mathbf{x})$, is expressed as

$$
\mathbf{v}^{1}(\mathbf{x})=\left(v_{1}^{1}(\mathbf{x}), v_{2}^{1}(\mathbf{x})\right)=-\frac{\nabla \Phi(\mathbf{x})}{\|\nabla \Phi(\mathbf{x})\|}, \mathbf{x} \in \Omega
$$

where $\Phi(\mathbf{x})$ satisfies the Eikonal equation $[5,6]$ 


$$
\left\{\begin{array}{c}
\|\Phi(\mathbf{x})\|=\frac{1}{U_{\max }}, \quad \mathbf{x} \in \Omega \\
\Phi(\mathbf{x})=0, \quad \mathbf{x} \in \Gamma_{o}
\end{array}\right.
$$

Here, $U_{\max }$ is the maximum walking speed of pedestrians.

2. Pedestrians display a tendency to avoid collisions with the environment (e.g., building walls and obstructions) while advancing towards their goal. The degree of tendency decreases if the distance to the pedestrian environment and the local pedestrian density increases. The vector normal to the environment, pointing inward, is $\mathbf{v}^{2}(\mathbf{x})=\left(v_{1}^{2}(\mathbf{x}), v_{2}^{2}(\mathbf{x})\right)$ with intensity

$$
\left(1-\frac{d(\mathbf{x})}{d_{\max }}\right)\left(1-\frac{\rho(\mathbf{x}, t)}{\rho_{\max }}\right) H\left(d_{\max }-d(\mathbf{x})\right) .
$$

Here, $d_{\max }$ is the safe distance that pedestrians prefer to keep from the environment, $d(\mathbf{x})$ is the distance from $\mathbf{x} \in \Omega$ to the borders of walls and obstacles, and $H(z)(z \in R)$ is the Heaviside step function (i.e. $H(z)=0$ where $z \leq 0$, and $H(z)=1$ where $z>0$ ).

3. Pedestrians tend to avoid the risk of collision among individuals, and then choose the less congested routes in order to feel comfortable. Therefore, they evaluate the crowd density by looking around within a certain visibility zone, and adjust the original direction of motion. The field of vision is defined as $R_{\Delta}(\mathbf{x})=\left[\theta_{0}-\Delta \theta, \theta_{0}+\Delta \theta\right]$ [7], where $\Delta \theta$ is the maximum visibility angle of pedestrians (in average), and

$$
\theta_{0}(\mathbf{x})=\arctan \left(\frac{v_{2}^{1}(\mathbf{x})}{v_{1}^{1}(\mathbf{x})}\right)
$$

is the angle that identifies the direction of the quickest path, $\mathbf{v}^{1}(\mathbf{x})$ at location $\mathbf{x} \in \Omega$. Let

$$
I=\left\{\boldsymbol{\mu}=(\cos (\alpha), \sin (\alpha)) \mid \alpha \in R_{\Delta}(\mathbf{x})\right\}
$$

be the vector set of the main direction of pedestrian motion. Thus, the direction of the minimum directional derivative, $\boldsymbol{v}^{3}(\mathbf{x}, t)$, is obtained as $\omega \boldsymbol{\mu}_{0}(\mathbf{x}, t)$, where $\boldsymbol{\mu}_{0}(\mathbf{x}, t)$ and the parameter $\omega$ are defined respectively as

$$
\min _{\boldsymbol{\mu} \in I} \nabla \rho(\mathbf{x}, t) \llbracket \boldsymbol{\mu} \text { and } \omega=\omega_{0}\left(\frac{\rho(\mathbf{x}, t)}{\rho_{\max }}\right)^{2}, \omega_{0} \geq 0
$$

Here, $\omega$ increases with the increasing local density, $\omega_{0}$ reflects the "comfort level" and $\rho_{\max }$ is the jam density.
According to the above hypotheses, the direction of pedestrian motion, $\boldsymbol{v}(\mathbf{x}, t)$, at position $\mathbf{x} \in \Omega$ at time $\mathrm{t}$, is obtained as

$$
\boldsymbol{v}(\mathbf{x}, t)=\frac{v^{1}(\mathbf{x})+v^{2}(\mathbf{x})+v^{3}(\mathbf{x}, t)}{\left\|v^{1}(\mathbf{x})+v^{2}(\mathbf{x})+v^{3}(\mathbf{x}, t)\right\|} .
$$

\section{NUMERICAL SIMULATION AND RESULTS}

The model is solved by a cell-centered high-resolution finite volume method for spatial discretization and a thirdorder fast sweeping method for an Eikonal equation on an orthogonal grid. A third-order total variation diminishing Runge-Kutta method is utilized for the time integration of the semi-discrete equation. See [8] for the detailed numerical algorithm.

The area of the walking facility is $100 \mathrm{~m} \times 50 \mathrm{~m}$ and its vertex on the underneath left side is taken as the coordinate origin. A square obstacle with sides of $20 \mathrm{~m}$ is located at $(50 \mathrm{~m}$, $20 \mathrm{~m})$. The entrance and exit are set at $x=0 m$ for $y \in[0 m, 50 m]$ and $x=100 m$ for $y \in[10 m, 40 m]$, respectively. At the initial time, the facility is empty, i.e. $\rho_{0}(\mathbf{x})=0$. The flux $\mathbf{F}(\mathbf{x}, t)=\rho(\mathbf{x}, t) \mathbf{v}(\mathbf{x}, t)$ at entrance $\Gamma_{i}$ is given by

$$
\mathbf{F}(\mathbf{x}, t)=\left\{\begin{array}{c}
(t / 30,0), 0 \leq t<60 \\
(4-t / 30), 0), 60 \leq t<120 \\
(0,0), 120 \leq t \leq 240
\end{array}\right.
$$

and $\mathbf{F}(\mathbf{x}, t)=0$ at the solid walls $\Gamma_{w}$. At exit $\Gamma_{0}$, the maximum outflow condition [9] is applied. The speed-density function is taken as $U_{e}(\rho)=U_{\max } e^{-\gamma\left(\rho / \rho_{\max }\right)^{2}}$ [10], with the parameters $U_{\max }=1.34 \mathrm{~m} / \mathrm{s}, \rho_{\max }=10 \mathrm{ped} / \mathrm{m}^{2}$, and $\gamma=7.5$. The critical density that divides the uncongested and congested density regions is $\rho_{c}=\rho_{\max } / \sqrt{2 \gamma} \approx 2.58 p e d / \mathrm{m}^{2}$. The maximum visibility angle of pedestrians, $\Delta \theta$, and the safe distance, $d_{\max }$, are set as $75^{\circ}$ and $1.6 \mathrm{~m}$, respectively.

Figure 1 shows the density distributions and flux vectors at different time steps for the dissipation of the crowd in four phases with the parameter $\omega_{0}=1$. In the first phase, the pedestrian flow is divided into two streams by the square obstacle and a triangular vacuum region is formed on the left side of the obstacle (see Fig. 1 (a)). In the second phase, two high-density regions that correspond to the traffic congestion (i.e. $\rho>\rho_{c}$ ) appear before the obstacle (see Fig. 1 (b)), because the width of the facility is reduced and pedestrians soon accumulate around the obstacle. At this time, small parts of the two streams merge on the right-hand side of the obstacle, leaving another triangular vacuum region in their wake, and some of the pedestrians have left the facility through the exit. In the third phase, the first triangular region 
disappears into a large empty region left by the departing crowd and pile-up phenomena caused by the crowd gathering and the relatively narrow exit can be seen at each end of the exit (see Fig. 1 (c)). In the fourth phase (see Fig. 1 (d)), most pedestrians have left the facility and others queue up around the exit due to its limited capacity.

To further illustrate the path-choice behaviors of pedestrians walking in the facility scattered an obstacle, various values of the "comfort level", $\omega_{0}$, are taken into account. In Fig. 2, we plot the pedestrian density along the lines, $x=35 m, x=95 m, y=5 m$ and $y=35 m$ at time $t=120 \mathrm{~s}$, with $\omega_{0}=0,0.5,1,2,5$, respectively. It is observed that the densities become smooth, when $\omega_{0}$ increases (see Fig. 2 (a)(d)). The "seeking comfort" behavior of pedestrians can prevent them from overly gathering around the obstacle and the exit. Therefore, this can significantly reduce the chance of traffic congestion occurring.

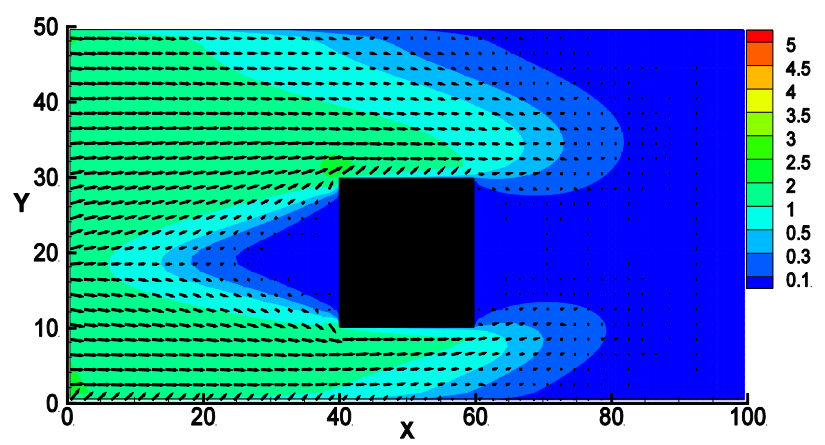

(a) $t=60 s$

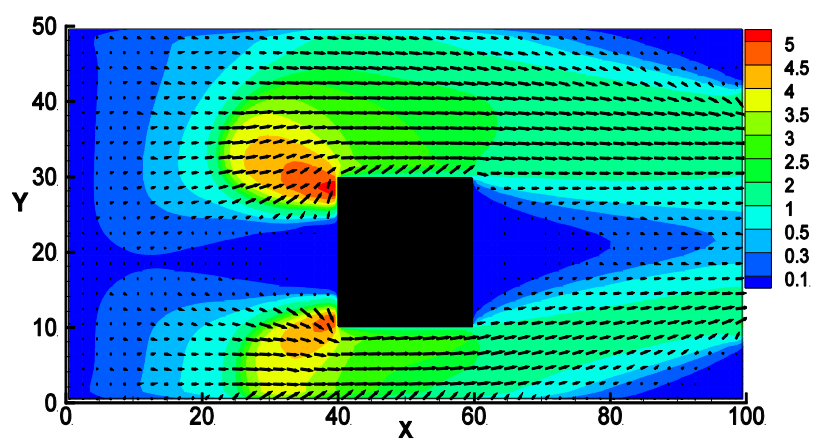

(b) $\mathrm{t}=120 \mathrm{~s}$

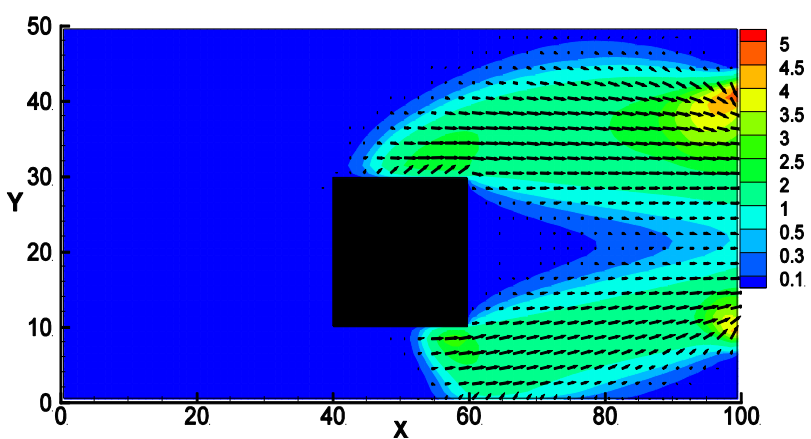

(c) $\mathrm{t}=180 \mathrm{~s}$

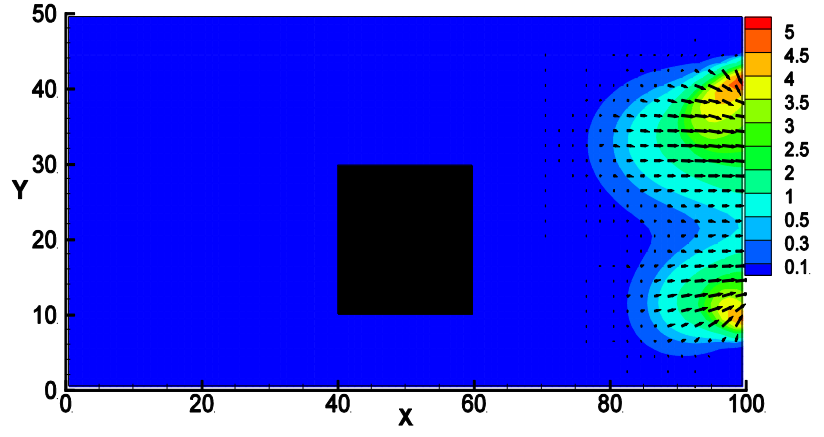

(d) $t=210 \mathrm{~s}$

Fig. 1. Pedestrian density $\rho$ and flux $\mathbf{F}$ at different times with $\omega_{0}=1$.

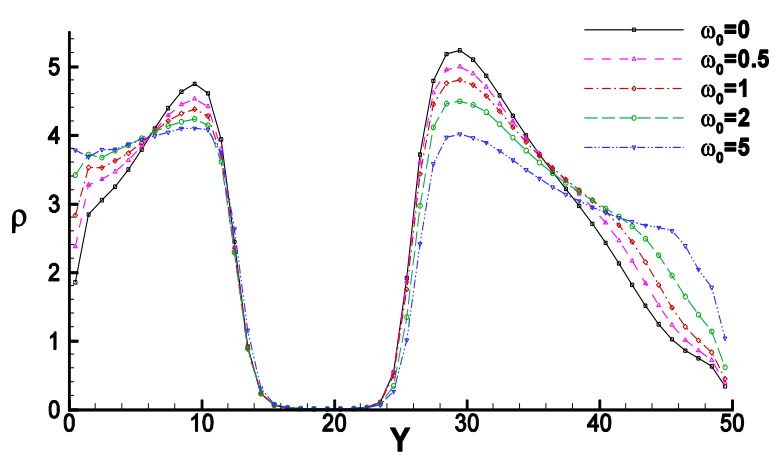

(a) $\mathrm{x}=35 \mathrm{~m}$

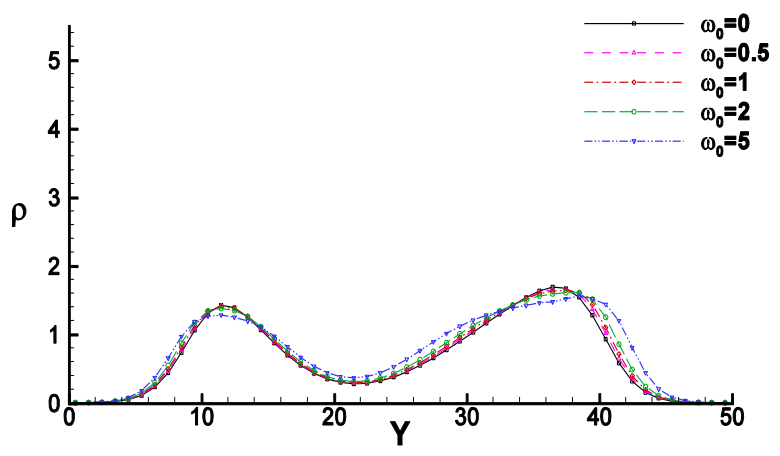

(b) $\mathrm{x}=95 \mathrm{~m}$

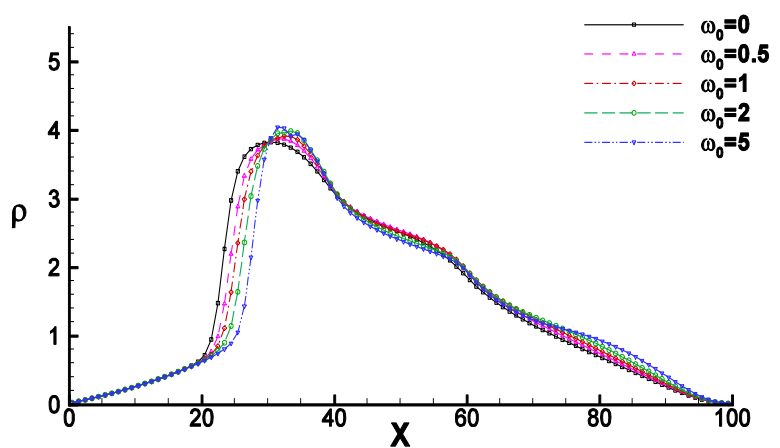

(c) $y=5 \mathrm{~m}$ 


\section{REFERENCES}

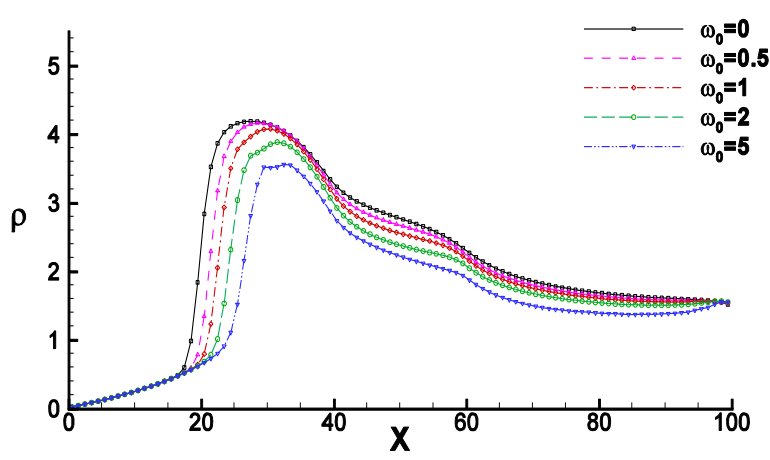

(d) $y=35 \mathrm{~m}$

Fig. 2. Pedestrian density $\rho$ along different lines at time $\mathrm{t}=120$ s, with $\omega_{0}=0,0.5,1,2,5$, respectively.

\section{CONCLUSIONS}

In this work, a macroscopic crowd model is developed to study pedestrian flow walking in a facility scattered with a square obstacle. Numerical results demonstrate that this model can reproduce some typical pedestrian flow characteristics and walking behaviors $[5,6]$, e.g. density distribution and pile-up phenomena. Further more, collision avoidance or "seeking comfort" behavior of pedestrians can significantly reduce the chance of traffic congestion occurring.

\section{ACKNOWLEDGMENT}

This work was supported by the Research Foundation of SWUST (No. 10zx7137), NSFC grant (No. 11202175) and NASF grant (No. 11076015).
[1] D. Helbing, I. Farkas, T. Vicsek, "Simulating dynamical features of escape panic", Nature 407, 487-490(2000).

[2] D. Helbing, "Traffic and related self-driven many-particle systems”, Rev. Mod. Phys. 73, 1067-1141(2001).

[3] M. Fukui, Y. Ishibashi, "Self-organized phase transitions in CAmodels for pedestrians", J. Phys. Soc. Japan 8, 2861-2863 (1999).

[4] A. Kirchner, A. Schadschneider, "Simulation of evacuation processes using a bionics-inspired cellular automation model for pedestrian dynamics", Physica A 312, 260-276(2002).

[5] L. Huang, S.C. Wong, M.P. Zhang, C.W. Shu, W.H.K. Lam, "Revisiting Hughes' dynamic continuum model for pedestrian flow and the development of an efficient solution algorithm". Trans Res Part B 43, 127-141(2009).

[6] Y.H. Xia, S.C. Wong, C.W. Shu, "Dynamic continuum pedestrian flow model with memory effect", Phys. Rev. E, 79, 066113 (2009).

[7] N. Bellomo, C. Dogbe, "On the modeling crowd dynamics from scaling to hyperbolic macroscopic models", Math. Mod. Meth. Appl. S. 18, 1317-1345(2008)

[8] Y.Q. Jiang, S.C. Wong, P. Zhang, R.X. Liu, Y.L. Duan, "Numerical simulation of a continuum model for bi-directional pedestrian flow", Appl. Math. Comput. 218, 6135-6143(2012).

[9] Y.Q. Jiang, S.C. Wong, P. Zhang, R.X. Liu, Y.L. Duan, "Numerical simulation of pedestrian flow past a circular obstruction", Acta Mech. Sin. 27, 215-221(2011).

[10] Y.Q. Jiang, P. Zhang, S.C. Wong, R.X. Liu, "A higher-order macroscopic model for pedestrian flows", Physica A, 389, 46234635(2010). 